\title{
Characterization of newly isolated oleaginous yeasts - Cryptococcus podzolicus, Trichosporon porosum and Pichia segobiensis
}

Ines Schulze*, Silla Hansen, Steffen Großhans, Thomas Rudszuck, Katrin Ochsenreither, Christoph Syldatk and Anke Neumann

\begin{abstract}
The yeast strains Cryptococcus podzolicus, Trichosporon porosum and Pichia segobiensis were isolated from soil samples and identified as oleaginous yeast strains beneficial for the establishment of microbial production processes for sustainable lipid production suitable for several industrial applications. When cultured in bioreactors with glucose as the sole carbon source C. podzolicus yielded $31.8 \%$ lipid per dry biomass at $20^{\circ} \mathrm{C}$, while $T$. porosum yielded $34.1 \%$ at $25^{\circ} \mathrm{C}$ and $P$. segobiensis $24.6 \%$ at $25^{\circ} \mathrm{C}$. These amounts correspond to lipid concentrations of 17.97 $\mathrm{g} / \mathrm{L}, 17.02 \mathrm{~g} / \mathrm{L}$ and $12.7 \mathrm{~g} / \mathrm{L}$ and volumetric productivities of $0.09 \mathrm{~g} / \mathrm{Lh}, 0.1 \mathrm{~g} / \mathrm{Lh}$ and $0.07 \mathrm{~g} / \mathrm{Lh}$, respectively. During the culture of C. podzolicus $30 \mathrm{~g} / \mathrm{l}$ gluconic acid was detected as by-product in the culture broth and $12 \mathrm{~g} / \mathrm{L}$ gluconic acid in T. porosum culture. The production of gluconic acid was eliminated for both strains when glucose was substituted by xylose as the carbon source. Using xylose lipid yields were $11.1 \mathrm{~g} / \mathrm{L}$ and $13.9 \mathrm{~g} / \mathrm{L}$, corresponding to $26.8 \%$ and $33.4 \%$ lipid per dry biomass and a volumetric productivity of $0.07 \mathrm{~g} / \mathrm{Lh}$ and $0.09 \mathrm{~g} / \mathrm{Lh}$, for C. podzolicus and T. porosum respectively. The fatty acid profile analysis showed that oleic acid was the main component (39.6 to 59.4\%) in all three strains and could be applicable for biodiesel production. Palmitic acid (18.4 to 21.1\%) and linolenic acid (7.5 to 18.7\%) are valuable for cosmetic applications. P. segobiensis had a considerable amount of palmitoleic acid (16\% content) and may be suitable for medical applications.
\end{abstract}

Keywords: Screening; Oleaginous yeasts; Microbial lipid production; Cryptococcus podzolicus; Trichosporon porosum; Pichia segobiensis

\section{Introduction}

As world population continues to grow, there is an everincreasing demand on energy and material resources. Therefore, to ensure long-term sustainability, suitable alternative production methods for oil as feedstock for several industrial applications have to be developed. Biodiesel and bioethanol derived from plant oil for example, have been used since time immemorial, but the disadvantage is the competition with the need to produce feed and food (Ratledge 1993). Therefore, oleaginous microorganisms represent an alternative production system for sustainable lipid production as they share the special feature to produce more than $20 \%$ lipid per dry biomass

\footnotetext{
* Correspondence: Ines.Schulze@kit.edu

Institute of Process Engineering in Life Sciences, Section II: Technical Biology, Karlsruhe Institute of Technology, Engler-Bunte-Ring 1, 76131 Karlsruhe, Germany
}

as carbon storage reserves with similar fatty acid compositions as plant oils.

Lipids are produced by all microorganisms (MOs) usually in the range of 6 to $8 \%$ per dry biomass principally as components for the cell membrane. However, oleaginous microorganisms, including yeasts, bacteria, filamentous fungi and microalgae, convert a carbon source when it is available in excess into intracellular triacylglycerols (TAG) as soon as a nitrogen limitation occurs (Ratledge 2002). These lipids are also called single cell oils (SCO) and are stored as lipid droplets within cells.

Baker's yeast Saccharomyces cerevisiae does not produce any intracellular lipid droplets (Vorapreeda et al. 2012), but several other yeast strains, however, are known to belong to the oleaginous microorganisms, e.g. Cryptococcus sp., Yarrowia sp., Candida sp., Rhodotorula sp., Rhodosporidium sp., Trichosporon porosum and

\section{黑 Springer}


Lipomyces sp. (Ratledge 1991). Cryptococcus curvatus is one of the most known oleaginous microorganisms able to grow on several carbon sources, e.g. glucose, glycerol or xylose (Meesters et al. 1996; Zhang et al. 2011). Advantages of microbial oil production compared to plant oil is the short life cycle of microbes and the possibility of an in vitro production process not influenced by external factors such as venue, season or climate (Thiru et al. 2011).

The yield and type of lipid is dependent on several factors including the type of microorganism, the culture conditions and the chosen substrates ( $\mathrm{Li}$ et al. 2008; Griffiths and Harrison 2009). Yeast strains produce mainly fatty acids which are similar in composition to those in plant oils containing predominantly saturated or monounsaturated fatty acids with carbon lengths of C16 and C18 (Papanikolaou and Aggelis 2011). Other microorganisms e.g. microalgae or filamentous fungi are also able to produce significant amounts of poly unsaturated fatty acids (PUFAs) like $\omega-3$ or $\omega-6$ fatty acids, e.g. docosahexaeonic acid (DHA) or eicosapentaenoic acid (EPA) (Ward and Singh 2005) which are usually extracted from fish oil. This knowledge permits the use of different microorganisms for different industrial applications.

Costs for microbial lipid production include the cost of the raw materials (chosen substrates), costs of the fermentation process (monitoring, control, labour, operating costs) and downstream processing costs. Fermentation costs are almost unchangeable. Substrate costs can be reduced by using low-cost substrates or waste material as carbon and nitrogen source. The greatest challenge for the downstream processing of intracellular lipid is overcoming the high energy costs for the cell disruption.

The establishment of an economic production process entails either attaining higher volumetric output of lipid or producing lipids with high value fatty acids. Therefore suitable microorganisms have to be identified or new strains have to be isolated from the environment which are able to grow on low-cost substrates, e.g. hemicellulosic wastes which include xylose as monomers.

Glucose can be converted by almost every microorganism. Therefore, it is suitable for screening experiments. However, many microorganisms, e.g. Pichia pastoris (Lee et al. 1986) are able to convert xylanes, a constituent of hemicelluloses and polymer of xylose molecules. Hemicellulosic material is accessible in large quantities all over the world (Pan et al. 2009) and can therefore be classified as low-cost substrate suitable as carbon source for oleaginous microorganisms. Soil samples contain old wood; hence products of decomposition like xylanes might be biotransformed by several soil microbes. Some of those microbes might belong to the group of oleaginous microorganisms showing the ability to convert xylose as carbon source into SCO. New isolates from soil samples may therefore be qualified for the application in biotechnological processes using hemicellulosic feedstock as cost-efficient substrate.

This study aimed to identify new yeast isolates from soil samples for the production of SCO, which can be used as oil substitute for industrial applications in the cosmetic, pharmaceutical, nutritional or energy sectors. Furthermore, xylose as low-cost substrate should be examined and evaluated as carbon source for promising lipid producing isolates.

\section{Material and methods \\ Microorganisms}

The oleaginous yeast Cryptococcus curvatus (ATCC 20509) was used as a positive control for SCO production. Saccharomyces cerevisiae (DSM 11285) as a non oleaginous yeast was taken as the negative control for yeasts not able to produce SCO.

Characterized soil isolates used in this study were deposited at the DSMZ culture collection. CPOH4 Cryptococcus podzolicus as DSM 27192, SSOH12 Pichia segobiensis as DSM 27193 and TPST6 Trichosporum porosum as DSM 27194.

\section{Soil sample collection}

Two samples were taken in summer time from peat bog soil in Kaltenbronn near Bad Wildbad in the black forest of Germany (sample 1: $48.720^{\circ} \mathrm{N}, 8.471^{\circ} \mathrm{E}, 894.4 \mathrm{~m}$ above sea level; sample 2: $48.716^{\circ} \mathrm{N}, 8.456^{\circ} \mathrm{E}, 911.8 \mathrm{~m}$ above sea level) and one soil sample was taken in summer time from a grassland in Karlsruhe (Baden-Württemberg, Germany, $48.98989^{\circ} \mathrm{N}, 8.40462^{\circ} \mathrm{E}, 116.7 \mathrm{~m}$ above sea level). All samples were taken at a depth of $5 \mathrm{~cm}$. The soil samples were stored at $-20^{\circ} \mathrm{C}$.

\section{Yeast isolation from soil samples}

A fraction of the collected soil sample $(10 \mathrm{mg})$ was resuspended in $1 \mathrm{ml}$ sterile demineralised water. An aliquot $(100 \mu \mathrm{l})$ of the suspension was plated out on YM agar plates (3 g/L yeast extract, $3 \mathrm{~g} / \mathrm{L}$ malt extract, $5 \mathrm{~g} / \mathrm{l}$ peptone, $10 \mathrm{~g} / \mathrm{L}$ glucose, $20 \mathrm{~g} / \mathrm{L}$ agar, $\mathrm{pH}$ 7) containing antibiotic (10 mg/L ampicillin, $20 \mathrm{mg} / \mathrm{L}$ tetracycline). Agar plates were incubated at $20^{\circ} \mathrm{C}$ until $1 \mathrm{~mm}$ diameter colonies became visible. Each colony was picked and viewed under a microscope to determine if it was a yeast. For long-term storage the isolated strains were stored in glycerol stocks $(15 \% \mathrm{w} / \mathrm{w})$ at $-80^{\circ} \mathrm{C}$.

\section{Screening for oleaginous microorganisms with Sudan Black B staining}

Isolated yeasts were cultivated on YM agar plates for 4 days at $20^{\circ} \mathrm{C}$. Replica plates were prepared by transferring the colonies from the original agar plate to a round filter paper (size of agar plate, GE Healthcare Europe 
GmbH, Freiburg, Germany, Whatman; Ref No 10311610). The filter paper was dried for $15 \mathrm{~min}$ at $60^{\circ} \mathrm{C}$ and subsequently stained for 20 min with $0.08 \%$ Sudan Black B in 96\%-Ethanol (EtOH) solution under shaking. Afterwards the filter was washed twice for 5 min with 96\% EtOH under shaking. Colonies which were stained blue could be potential oleaginous MOs with intracellular TAGs (Evans et al. 1985)

\section{Identification of the isolates}

Genomic DNA was isolated using the commercial kit "Precellys Bacterial/ Fungal DNA-Kit" (PEQLAB Biotechnologie $\mathrm{GmbH}$, Erlangen, Germany; 12-7511-00). Afterwards polymerase-chain reaction (PCR) fragments were produced applying universal primers ITS1 (5'-TC CGTAGGTGAACCTGCG-3') (Eurofins MWG GmbH, Ebersberg, Germany) and ITS4 (5'-TCCTCCGCTTATTGA TATGC-3') (Eurofins MWG GmbH, Ebersberg, Germany) (Fujita et al. 2001). PCR amplification was performed in a total volume of $50 \mu \mathrm{l}$. The composition of each PCR reaction was as followed: $5 \mu$ PCR buffer (Dream Taq Green buffer, Thermo Scientific Fermentas, Schwerte, Germany; \#B71), $5 \mu \mathrm{l}$ of dNTP mixture (2 mM each) (Thermo Scientific Fermentas, Schwerte, Germany; \#R0241), $1 \mu \mathrm{l}$ ITS1 primer $(10 \mu \mathrm{M}), 1 \mu \mathrm{l}$ ITS4 primer $(10 \mu \mathrm{M})$ and $0.5 \mu \mathrm{l}$ Dream Taq DNA polymerase (Thermo Scientific Fermentas, Schwerte, Germany; EP0701) were filled up with PCR water (Carl ROTH GmbH, Karlsruhe, Germany; T143.4). The PCR amplification started with $95^{\circ} \mathrm{C}$ for initial denaturation, followed by 30 cycles of denaturation at $95^{\circ} \mathrm{C}$ for $30 \mathrm{~s}$, annealing at $48^{\circ} \mathrm{C}$ for $30 \mathrm{~s}$, and extension at $72^{\circ} \mathrm{C}$ for $1 \mathrm{~min}$. The final extension was done at $72^{\circ} \mathrm{C}$ for $10 \mathrm{~min}$. PCR products were visualized on $1 \%$ agarose gel (1× TAE-buffer: $40 \mathrm{mM}$ Tris base, 1 mM EDTA, pH 8 adjusted with acetic acid; $0.1 \mu \mathrm{g} / \mathrm{ml}$ ethidium bromide) after carrying out gel electrophoresis of each PCR amplification product and $6 \mu \mathrm{l}$ Quick Load $1 \mathrm{~kb}$ DNA Ladder (New England Biolabs, Frankfurt/Main, Germany; N0468 S) with $1 \times$ TAE buffer at $100 \mathrm{~V}$ for $1 \mathrm{~h}$. Distilled water was used as negative control. The amplified DNA (including the $5.8 \mathrm{~S}$ rDNA) was sequenced by GATC Biotech Corporation (Konstanz, Germany). Alignments were performed via MEGABLAST with NCBI database (http:// www.ncbi.nlm.nih.gov/).

\section{Cultivation in shake flasks}

YM medium (3 g/L yeast extract, $3 \mathrm{~g} / \mathrm{L}$ malt extract, $5 \mathrm{~g} / \mathrm{l}$ peptone, $\mathrm{pH} 7$ ) was supplemented with glucose to an initial concentration of $50 \mathrm{~g} / \mathrm{L}$ glucose. $50 \mathrm{ml}$ initial culture volume filled in $500 \mathrm{ml}$ conical shake flasks with an initial optical density $\left(\mathrm{OD}_{600}\right)$ of 0.5 were incubated at $130 \mathrm{rpm}$ at $25^{\circ} \mathrm{C}$ for 120 hours. Every 24 hours $35 \mathrm{~g} / \mathrm{L}$ glucose was repeatedly added to ensure that the carbon source was in excess.

\section{Cultivation in bioreactors}

For the cultivation in the bioreactor a mineral salt medium was used, formulated on a phosphate buffer at pH 5 (8.99 g/L $\mathrm{KH}_{2} \mathrm{PO}_{4}$ and $\left.0.12 \mathrm{~g} / \mathrm{L} \mathrm{Na}_{2} \mathrm{HPO}_{4} * 2 \mathrm{H}_{2} \mathrm{O}\right)$ which was based on the medium used in Meesters et al. (1996). The medium constituents were $0.1 \mathrm{~g} / \mathrm{L}$ sodium citrate $2 \mathrm{H}_{2} \mathrm{O}, 0.1 \mathrm{~g} / \mathrm{L}$ yeast extract, $0.2 \mathrm{~g} / \mathrm{L} \mathrm{MgSO}_{4} * 7 \mathrm{H}_{2} \mathrm{O}$, $4.72 \mathrm{~g} / \mathrm{L}\left(\mathrm{NH}_{4}\right)_{2} \mathrm{SO}_{4}$ (refers to $\left.1 \mathrm{~g} / \mathrm{L} \mathrm{N}\right)$. Once a day the culture broth was supplemented with $2 \mathrm{ml}$ trace elements solution $\left(4 \mathrm{~g} / \mathrm{L} \quad \mathrm{CaCl}_{2} * 2 \mathrm{H}_{2} \mathrm{O}, 0.55 \mathrm{~g} / \mathrm{L} \quad \mathrm{FeSO}_{4} * 7 \mathrm{H}_{2} \mathrm{O}\right.$, $0.475 \mathrm{~g} / \mathrm{L}$ citric acid, $0.1 \mathrm{~g} / \mathrm{L} \mathrm{ZnSO}_{4} * 7 \mathrm{H}_{2} \mathrm{O}, 0.076 \mathrm{~g} / \mathrm{L}$ $\mathrm{MnSO}_{4}{ }^{*} \mathrm{H}_{2} \mathrm{O}, 100 \mu \mathrm{l} 18 \mathrm{M} \mathrm{H}_{2} \mathrm{SO}_{4}$ ) and $2 \mathrm{ml}$ salt solution $\left(20 \mathrm{~g} / \mathrm{L} \mathrm{MgSO}_{4} * 7 \mathrm{H}_{2} \mathrm{O}, 10 \mathrm{~g} / \mathrm{L}\right.$ yeast extract) per $100 \mathrm{ml}$ cultivation medium. Precultures were prepared in conical shake flasks with initial $\mathrm{OD}_{600}$ of 0.5 and $120 \mathrm{rpm}$. Fermentation was performed in a $2.5 \mathrm{~L}$ fermentor (Infors HT, Bottmingen, Switzerland; Minifors fermentor) with $1 \mathrm{~L}$ initial culture medium, initial $\mathrm{OD}_{600}$ of 1 , at $600 \mathrm{rpm}$ and with 1 vvm aeration rate without control of dissolved oxygen $\left(\mathrm{pO}_{2}\right)$ for at least $188 \mathrm{~h}$. $\mathrm{CPOH} 4$ was grown at $20^{\circ} \mathrm{C}$, $\mathrm{SSOH} 12$ and TPST6 at $25^{\circ} \mathrm{C}$. The control of pH was done automatically by addition of $4 \mathrm{M} \mathrm{H}_{3} \mathrm{PO}_{4}$ and $4 \mathrm{M} \mathrm{NaOH}$ in each fermentor, Contraspum A 4050 HAC (Zschimmer und Schwarz GmbH und Co KG, Lahnstein, Germany) was applied as antifoam agent. Initial glucose or xylose concentration was $50 \mathrm{~g} / \mathrm{L}$. Each day the carbon source was replenished to a maximum concentration of $90 \mathrm{~g} / \mathrm{L}$ after determining the actual concentration. A minimum of five samples were taken per day (four samples à $3 \mathrm{ml}$ for the determination of $\mathrm{OD}_{600}$, dry biomass $(\mathrm{g} / \mathrm{L})$, carbon and nitrogen source and by-products; one sample à $20 \mathrm{ml}$ for lipid analysis (\% lipid/dry biomass) via gas chromatography).

\section{Sample preparation for dry biomass and analysis of supernatant}

Dry biomass was analyzed gravimetrically. A $1 \mathrm{ml}$ aliquot of the culture fluid was transferred into a predried and preweighed $1.5 \mathrm{ml}$ reaction tube and centrifuged at 13,000 rpm for $5 \mathrm{~min}$. The supernatant was collected and used for the determination of glucose and $\mathrm{NH}_{4}^{+}$. The cell pellet was washed with $800 \mu \mathrm{l}$ saline $(0.9 \% \mathrm{NaCl})$, dried at $60^{\circ} \mathrm{C}$ for $24 \mathrm{~h}$ and weighed.

Analysis of $\mathrm{NH}_{4}^{+}$, glucose, xylose, ethanol, gluconic acid All components were measured in triplicates with enzymatic test kits. D-Glucose (10716251035), ethanol (10176290035) and gluconic acid (10428191035) were purchased at R-Biopharm AG (Darmstadt Germany). $\mathrm{NH}_{4}^{+}$was measured via Spectroquant kit (Merck KGaA, Darmstadt, Germany 1.14752.0001). D-Xylose assay kit was taken for the concentration of xylose (Megazyme, Bray, Ireland; K-XYLOSE). 


\section{HPLC analysis of organic acids}

The pure supernatant was taken to measure five different organic acids (gluconic, malic, citric, succinic and fumaric acid) using a standard HPLC device (Agilent 1100 Series, Agilent Technologies Deutschland $\mathrm{GmbH}$, Böblingen, Germany) equipped with a $150 \times 4.6 \mathrm{~mm}$ HPLC column Synergi ${ }^{\mathrm{Tm}} 4 \mu \mathrm{m}$ Fusion-RP $80 \AA$ (Phenomenex, Aschaffenburg, Germany; 00 F-4424-E0) at $30^{\circ} \mathrm{C}$

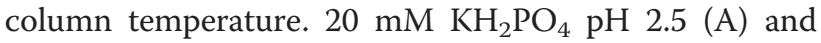
$100 \%$ Methanol (B) were used as eluents to drive the following temporal gradients. 0-0.5 min 100\% eluent A, $0.5-10$ min increase of eluent B from $0 \%$ to $10 \%$, 10-12 min decrease of eluent B from $10 \%$ back to $0 \%$ and $12-14$ min again $100 \%$ eluent A. $10 \mu \mathrm{L}$ sample was injected, a flow rate of $1 \mathrm{ml} / \mathrm{min}$ was adjusted and peaks were detected via UV at $220 \mathrm{~nm}$.

\section{Lipid analysis}

A $20 \mathrm{ml}$ aliquot of the culture broth was centrifuged $(4,700 \mathrm{rpm} 5 \mathrm{~min})$, the pellet was resuspended in saline $(0.9 \% \mathrm{NaCl})$ and again centrifuged (4,700 rpm, $5 \mathrm{~min})$. The supernatant was discarded and the pellet was freeze dried $\left(-30^{\circ} \mathrm{C}, 0,370 \mathrm{mbar}\right)$. Preparation for the quantitative and qualitative gas chromatographical analysis was done in a one-step-procedure by direct esterification plus extraction. A portion $(20 \mathrm{mg})$ of freeze dried biomass was weighed into a $15 \mathrm{ml}$ glass falcon with teflon cap. $1.5 \mathrm{ml}$ hexane and $0.5 \mathrm{ml}$ of $2 \mathrm{mg} / \mathrm{ml}$ internal standard (methyl benzoate) dissolved in hexane were added as solvent for the extraction of lipid. In addition, $2 \mathrm{ml} \mathrm{15 \%} \mathrm{H}_{2} \mathrm{SO}_{4}$ in methanol was added for the esterification step. Each sample was heated up to $100^{\circ} \mathrm{C}$ for $2 \mathrm{~h}$ with continuous shaking. After cooling on ice, $1 \mathrm{ml}$ demineralised water was added. The mixture was centrifuged for $5 \mathrm{~min}$ at 2,500 rpm. $1 \mu \mathrm{l}$ of the upper phase, containing the fatty acid methyl esters extract, was analyzed via chromatography (Agilent Technologies, 6890 N Network GC-System). The instrument was equipped with a DBWax column (l: $30 \mathrm{~m} \mathrm{~d}$ : $0.25 \mathrm{~mm}$, Agilent Technologies Deutschland GmbH, Böblingen, Germany; 122-7032), a flame ionization detector and working with a pressure of 1.083 bar and initial temperature of $40^{\circ} \mathrm{C}$. The column temperature was increased from $40^{\circ} \mathrm{C}$ to $250^{\circ} \mathrm{C}$ with a rate of $8^{\circ} \mathrm{C} / \mathrm{min}$. The temperature was held at $250^{\circ} \mathrm{C}$ for $10 \mathrm{~min}$ before cooling down to $40^{\circ} \mathrm{C}$. The total fatty acid content and the identification of fatty acids were performed using the standard RM3 FAME Mix (Sigma Aldrich, Taufkirchen, Germany; 07256-1AMP) and Marine FAME Mix (Acid Methyl Ester Marine Oil FAME Mix) (Restek GmbH, Bad Homburg, Germany; 35066). Fatty acids which represented less than $1 \%$ of total fatty acids were combined to "trace fatty acids".

\section{Accession numbers}

The following EMBL accession numbers have been assigned for the fungal ribosomal genes of the isolates:
CPOH4 Cryptococcus podzolicus as HG737350, SSOH12 Pichia segobiensis as HG737349 and TPST6 Trichosporum porosum as HG737348.

\section{Results}

Screening and identification of yeast isolates

Four different yeast strains in total could be isolated from three soil samples. Three isolates arose from peat bog samples and one isolate (TPST6) from grass land soil sample. Peat bog soil is assumed to contain a high level of carbon compared to nitrogen. Three out of those four yeast isolates were identified as potential lipid producers using the Sudan Black B staining technique on agar plates supplemented with antibiotic. The sequences of ITS region were compared with the nucleotide data base using the NCBI-blast tool. SSOH12 showed highest genetic agreement with Pichia segobiensis closely followed by Scheffersomyces stipitis which is a synonym of Pichia stipitis. CPOH4 was identified as Cryptococcus podzolicus. TPST6 was identified as Trichosporon porosum. The results are presented in Table 1. The fourth isolated yeast which could not be identified as oleaginous showed highest agreement to Candida shehatae, but was not further investigated in this study.

\section{Shake flask cultivation}

All isolates were cultivated in $500 \mathrm{ml}$ shake flasks containing YM medium supplemented with $50 \mathrm{~g} / \mathrm{L}$ glucose. The flasks were incubated at $25^{\circ} \mathrm{C}$ for 120 hours. C. curvatus, a well known oleaginous yeast, was taken as the positive control and S. cerevisiae as the negative control. Glucose was taken as standard carbon source and was held in excess throughout the fermentation period. The $\mathrm{pH}$ value did not decrease below 4 in all cultivations. The results are presented in Figure 1. As expected, the oleaginous yeast C. curvatus yielded more than $40 \%$ lipid per dry biomass whereas $S$. cerevisiae, a typical non-oleaginous microorganism, yielded less than $10 \%$ lipid per dry biomass corresponding to the normal cellular amount of lipids.

The best lipid producer among the three new isolates was $\mathrm{CPOH} 4$ with $34.6 \%$ lipid per dry biomass and can therefore be classified as oleaginous under the chosen conditions in YM medium with additional glucose and shake flasks. TPST6 yielded 24.5\% lipid per dry biomass, hence this yeast also belongs to the oleaginous microorganisms. However, SSOH12 yielded $11.2 \%$ lipid per dry biomass; hence SSOH12 cannot be classified as lipid producing strain under the chosen conditions in shake flasks with YM medium with additional glucose.

\section{Cultivation of the three isolated yeasts in $2.5 \mathrm{~L}$-fermentor on glucose or xylose}

All three isolates SSOH12, CPOH4 and TPST6 were cultivated in bioreactors at $\mathrm{pH} 5$ and $600 \mathrm{rpm}$ during 
Table 1 Results of the sequencing of ITS region including 5.8 S rRNA of isolates SSOH12, CPOH4 and TPST6

\begin{tabular}{lllllll}
\hline Isolate & $\begin{array}{l}\text { Deposited at DSMZ as } \\
\text { (EMBL accession number) }\end{array}$ & Total score & Query coverage [\%] & E-value & Max identity & $\begin{array}{l}\text { Closest relative in NCBI } \\
\text { data bank (accession number) }\end{array}$ \\
\hline SSOH12 & DSM 27193 (HG737349) & 1062 & 99 & 0 & 99 & Pichia segobiensis (DQ409166.1) \\
SSOH12 & DSM 27193 (HG737349) & 1059 & 99 & 0 & 99 & Scheffersomyces stipitis (JQ026363.1) \\
CPOH4 & DSM 27192 (HG737350) & 852 & 100 & 0 & 100 & Cryptococcus podzolicus (HF558652.1) \\
TPST6 & DSM 27194 (HG737348) & 878 & 100 & 0 & 100 & Trichosporon porosum (HF558656.1) \\
\hline
\end{tabular}

DSMZ: German Collection of Microorganisms and Cell Cultures, EMBL: The European molecular Biology Laboratory, NCBI: National Center for Biotechnology Information.

the whole cultivation. Hence, compared to shake flask cultivation a constant $\mathrm{pH}$ and higher aeration rates were ensured. $\mathrm{CPOH} 4$ was cultivated at $20^{\circ} \mathrm{C}$ in preliminary studies (data not shown) revealed best growth at this temperature. SSOH12 and TPST6 were cultivated at $25^{\circ} \mathrm{C}$ as this was the suggested cultivation temperature for T. porosum (Middelhoven et al. 2001) and described growth conditions for $S$. segobiensis at ATCC data bank. The carbon source, glucose or xylose, was held in excess throughout the fermentation, but less than $90 \mathrm{~g} / \mathrm{L}$ to prevent substrate inhibition. All strains were cultivated on glucose as the carbon source, but $\mathrm{CPOH} 4$ and TPST6 were also grown on xylose. Table 2 summarizes the main results of the different fermentations; growth yield coefficient $\left(y_{x / s}\right)$, product yield coefficient $\left(y_{p / s}\right)$, volumetric productivity $\left(\mathrm{Q}_{\mathrm{L}}\right)$, lipid content per dry biomass (\%lipid/dry biomass), lipid concentration (g/L) and detected by-products are presented. The data for $\mathrm{CPOH} 4$ on glucose and xylose in Table 2 represent the mean from two independent fermentations. The data for TPST6 on glucose and xylose as well as the data for SSOH12 on glucose are single fermentations. To illustrate the lipid production processes in more detail the fermentations of all three isolates are exemplarily shown for one single fermentation in Figures 2, 3, 4, 5 and 6. Due to daily feeding of the carbon source in the case of $\mathrm{CPOH} 4$ and SSOH12 the values for the dry biomass are shown before and after feeding. Concerning TPST6, feeding of the carbon source was repeated only two times after 26 and $48 \mathrm{~h}$. A third feeding was performed after $94 \mathrm{~h}$ only for the cultivation with glucose. For all isolates the lipid production started as soon as the nitrogen source $\mathrm{NH}_{4}^{+}$was totally consumed (approximately after 50 hours). The dissolved oxygen level reached minimum values of $0 \%$ during the maximal growth phase, but increased again as soon as the dry biomass started to stagnate. All three yeast isolates exceeded a lipid content of more than $20 \%$ lipid per dry biomass (Table 2). The highest lipid content was reached with yeast isolate TPST6. Using glucose as carbon source this strain produced up to $33.4 \%$ lipid per dry biomass, followed by $\mathrm{CPOH} 4$ (31.8\%) and SSOH12 (24.6\%). When cultured on xylose CPOH4 gave $26.8 \%$ lipid per dry biomass, whereas TPST6 yielded even $33.4 \%$. Under the chosen conditions of the fermentations all three isolates may be classified as oleaginous yeasts. The determination of by-products was carried out

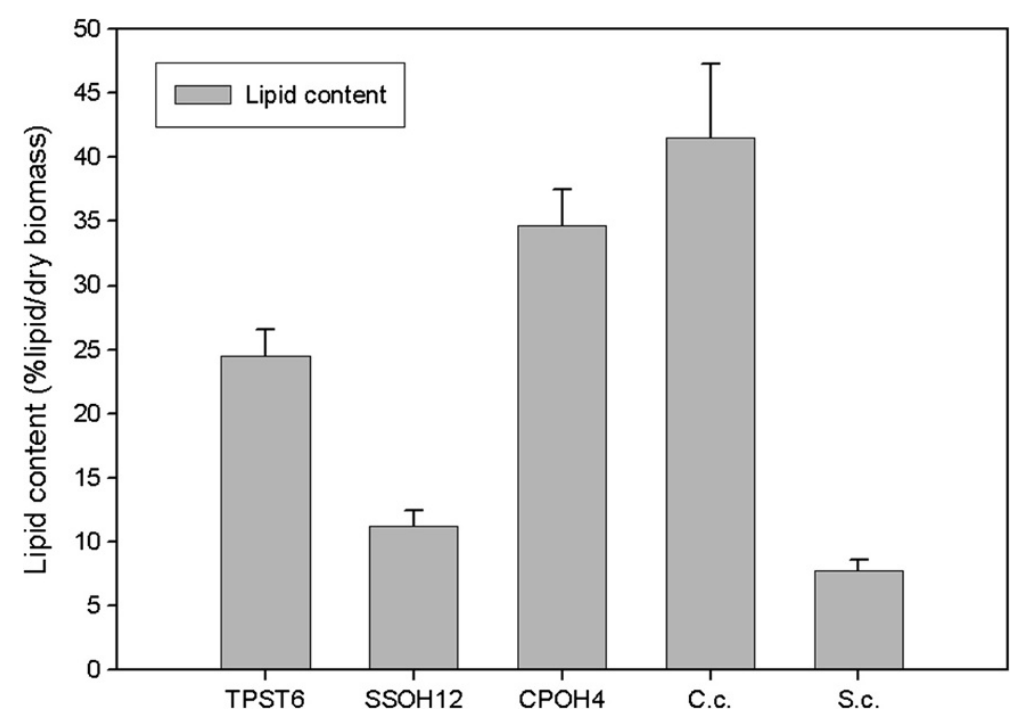

Figure 1 Lipid content in shake flask cultivation at $25^{\circ} \mathrm{C}$ in YM medium after $120 \mathrm{~h}$ of all four isolates TPST6, CSOH1, SSOH12 and $\mathrm{CPOH} 4$ as well as oleaginous yeast Cryptococcus curvatus (C.c.) and non oleaginous yeast Saccharomyces cerevisiae (S.c.). 
Table 2 Overview about performed fermentations of isolates SSOH12, CPOH4 and TPST6 at pH 5 in mineral salt medium; SSOH12 and TPST6 represent single fermentations, whereas CPOH4 are mean of two independent fermentations including standard deviations

\begin{tabular}{|c|c|c|c|c|c|}
\hline Isolate & SSOH12 & $\mathrm{CPOH} 4$ & & TPST6 & \\
\hline Carbon source & Glucose & Glucose & Xylose & Glucose & Xylose \\
\hline Process time $[\mathrm{h}]$ & 188 & 188 & & 161 & \\
\hline $\mathrm{T}\left[{ }^{\circ} \mathrm{C}\right]$ & 25 & 20 & & 25 & \\
\hline Dry biomass $_{\max }[\mathrm{g} / \mathrm{L}]$ & 51.7 & $56.5 \pm 5.2$ & $41.5 \pm 1.24$ & 49.9 & 41.5 \\
\hline $\mathrm{Y}_{\mathrm{x} / \mathrm{s}}[\mathrm{g} / \mathrm{g}]$ & 0.35 & $0.50 \pm 0.0$ & $0.53 \pm 0.02$ & 0.38 & 0.41 \\
\hline$Y_{p / s}[g / g]$ & 0.06 & $0.11 \pm 0.01$ & $0.09 \pm 0.0$ & 0.11 & 0.12 \\
\hline$Q_{L}\left[g / L^{*} h\right]$ & 0.07 & $0.09 \pm 0.04$ & $0.07 \pm 0.01$ & 0.1 & 0.09 \\
\hline Lipid content [\%lipid/dry biomass] & 24.6 & $31.8 \pm 8.0$ & $26.8 \pm 1.2$ & 34.1 & 33.4 \\
\hline Concentration of lipid [g/L] & 12.7 & 18 & 11.1 & 17 & 13.9 \\
\hline Gluconic acid [g/L] & n.d. & 30 & n.d. & 12 & n.d. \\
\hline Ethanol [g/L] & $>3.3$ & $<1.0$ & $<1.0$ & $<1.0$ & $<1.0$ \\
\hline
\end{tabular}

as there were some indications for ethanol and acid production. In the culture broth of SSOH12 ethanol was detected whereas ethanol production in the case of $\mathrm{CPOH} 4$ and TPST6 was negligible (Table 2). Instead, $\mathrm{CPOH} 4$ and TPST6 showed evidence of acid production when cultured on glucose. This acid was identified as gluconic acid by HPLC analysis and was confirmed with an enzymatic test. Malic, citric, succinic and fumaric acids were not detected. These acids were neither detected for SSOH12 cultured on glucose nor for $\mathrm{CPOH} 4$ and TPST6 cultured on xylose. Cultured on glucose, $\mathrm{CPOH} 4$ yielded the highest lipid concentration with $18.0 \mathrm{~g} / \mathrm{L}$ and the highest concentration of gluconic acid with $30 \mathrm{~g} / \mathrm{L}$, followed by TPST6 with a lipid concentration of $17.0 \mathrm{~g} / \mathrm{L}$ and $12 \mathrm{~g} / \mathrm{L}$ gluconic acid. SSOH12 resulted only in $12.7 \mathrm{~g} / \mathrm{L}$ lipid and a minimum of $3.3 \mathrm{~g} / \mathrm{L}$ ethanol as by-product. Considering the lipid productivity $\left(\mathrm{Q}_{\mathrm{L}}\right)$, TPST6 grown on glucose may be the best lipid producer $(0.10 \mathrm{~g} / \mathrm{Lh})$ followed by TPST 6 on $\mathrm{xy}$ lose and $\mathrm{CPOH} 4$ on glucose with the same value of $0.09 \mathrm{~g} / \mathrm{Lh}$.

\section{Fatty acid profile}

The analysis of the fatty acid profile (Table 3 ) revealed different profiles for each isolate. The main fatty acid in all isolates was oleic acid (C18:1) with content ranging between $39.6 \%$ and $59.4 \%$. Relatively high yields of palmitic acid (C16:0) ranging from $18.4 \%$ to $21.1 \%$ were obtained in all three isolates. In addition to oleic and palmitic acid, palmitoleic acid (C16:1) with $16 \%$ was one of the main products in $\mathrm{SSOH} 12$ whereas $\mathrm{CPOH} 4$ and TPST6 produced only negligible amounts of this fatty acid. In contrast, TPST6 was characterized by high

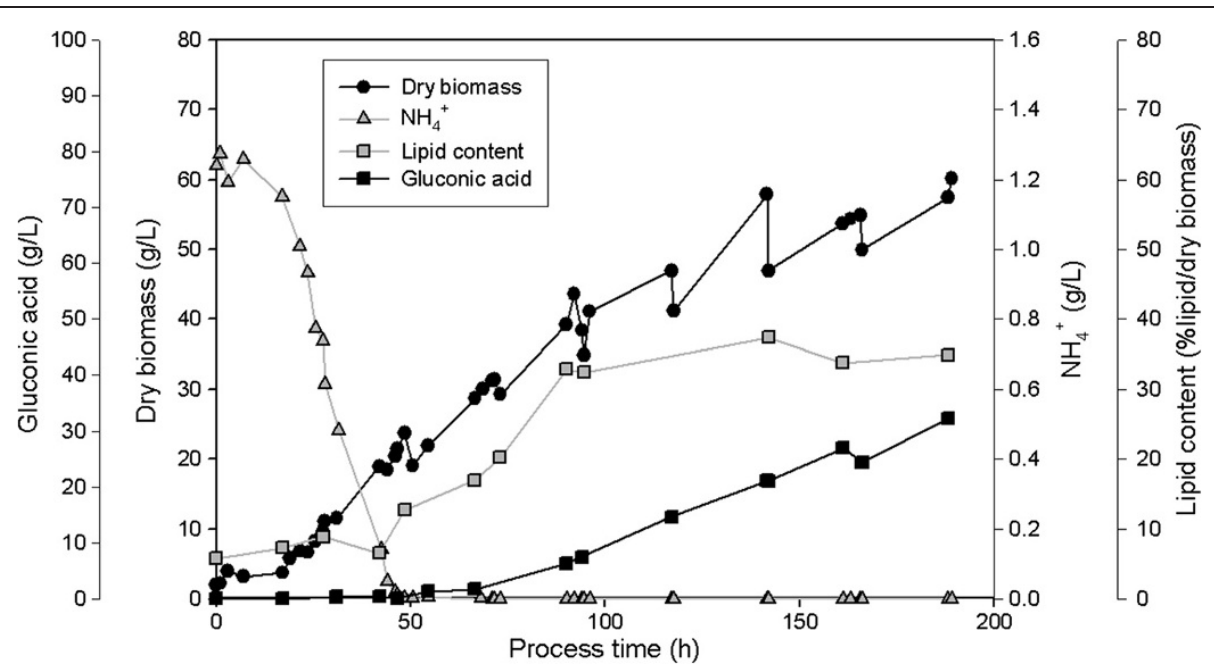

Figure $2 \mathrm{CPOH} 4$ cultivated on glucose in $2.5 \mathrm{~L}$-bioreactor in mineral salt medium at $\mathrm{pH} 5$ and $20^{\circ} \mathrm{C}$; glucose was fed daily; $\mathrm{NH}_{4}^{+}$ consumption, production of biomass, lipid and gluconic acid are presented. 


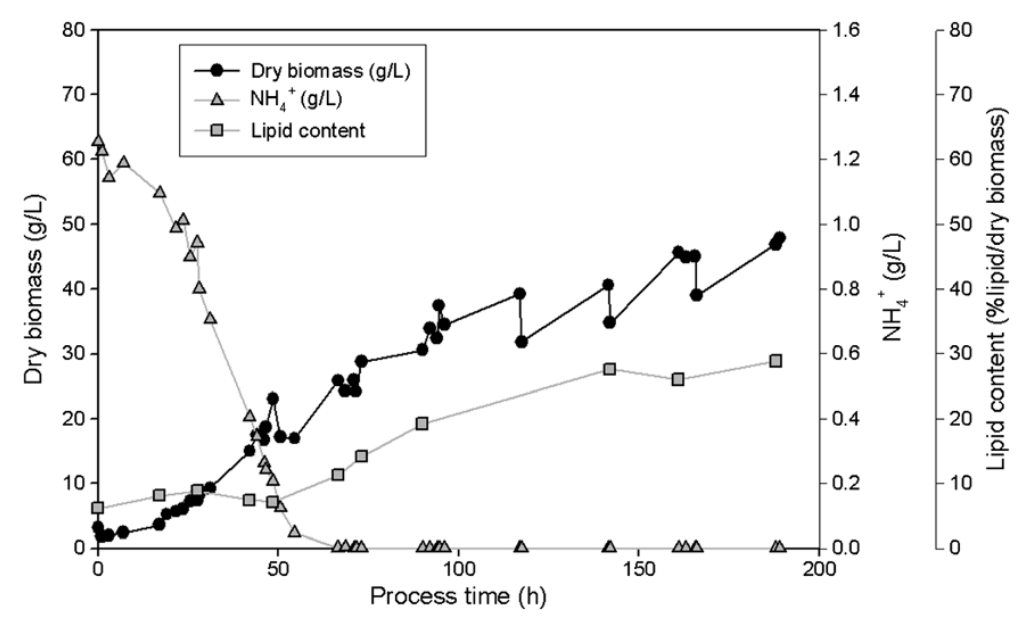

Figure $3 \mathrm{CPOH} 4$ cultivated on xylose in $2.5 \mathrm{~L}$-bioreactor in mineral salt medium at $\mathrm{pH} 5$ and $20^{\circ} \mathrm{C}$; xylose was fed daily.

$\mathrm{NH}_{4}^{+}$consumption, production of biomass and lipid are presented.

amounts of stearic acid (C18:0) and linolic acid (C18:2) ranging between $15.5 \%$ and $18.7 \%$. $\mathrm{CPOH} 4$ produced $5 \%$ of stearic acid (C18:0) and just 8.7 to $11.1 \%$ of linolic (C18:2) acid. The amount of linolenic acid (C18:3) was less than $1.3 \%$ and is therefore negligible in all isolates.

There was no remarkable difference in most of the fatty acid profile comparing glucose and xylose as the carbon source. However, for both isolates $\mathrm{CPOH} 4$ and TPST6 a slightly higher amount of oleic acid (C18:1) and slightly lower amount of linolic acid (C18:2) was observed when cultured on glucose.

\section{Discussion}

Three new oleaginous yeast strains were isolated from soil samples arising from a peat bog and grassland using
Sudan Black B staining on agar plates. The reason for peat bog soil was the fact that it contains high amount of carbon arising from old wood and plants while nitrogen content is low. The use of a grassland sample was chosen as a comparison sample without any specific limitations.

The first strain SSOH12 was identified as Pichia segobiensis producing a considerable amount of $16 \%$ palmitoleic acid (C16:1) of total lipid amount. The second strain $\mathrm{CPOH} 4$ was identified as Cryptococcus podzolicus and yielded the highest lipid concentration with $18.0 \mathrm{~g} / \mathrm{L}$ of all isolates. The third strain TPST6 was identified as Trichosporon porosum and produced a considerable amount of $17.8 \%$ linolic acid (C18:2) on glucose and 18.7\% on xylose. All three strains have not been described as

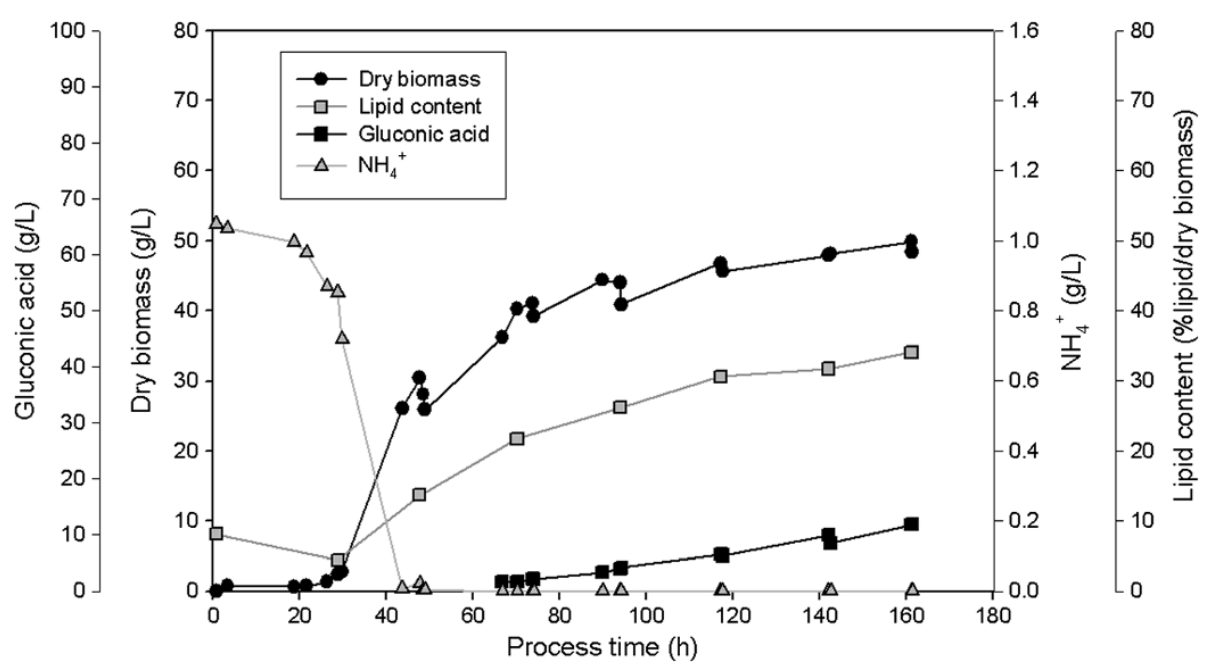

Figure 4 TPST6 cultivated on glucose in 2.5 L-bioreactor in mineral salt medium at pH 5 and $25^{\circ} \mathrm{C}$; glucose was fed daily. $\mathrm{NH}_{4}^{+}$consumption, production of biomass, lipid and gluconic acid are presented. 


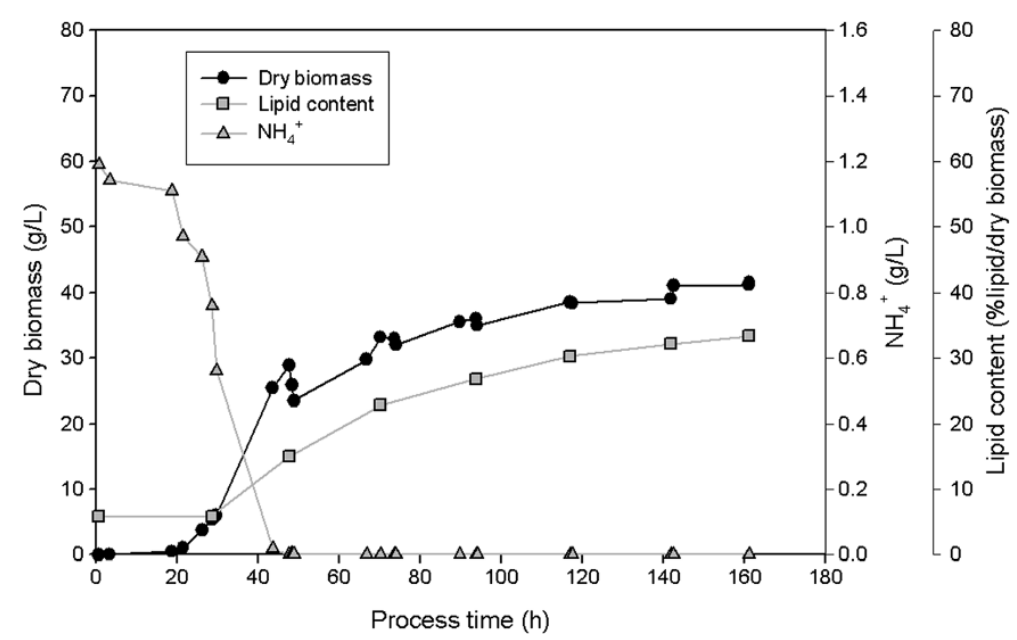

Figure 5 TPST6 cultivated on xylose in 2.5 L-bioreactor in mineral salt medium at $\mathrm{pH} 5$ and $25^{\circ} \mathrm{C}$; xylose was fed daily.

$\mathrm{NH}_{4}^{+}$consumption, production of biomass and lipid are presented.

oleaginous before. Due to sufficient production of lipid amounts and interesting fatty acid profiles, further studies of all three strains are worthwhile to establish sustainable bioprocesses for the production of adequate amounts of microbial oil for industrial applications.

\section{Influence of cultivation conditions on lipid production for screening experiments}

Different lipid contents per dry biomass were reached depending on the manner of cultivation, if choosing a shake flask or a bioreactor. C. podzolicus $\mathrm{CPOH} 4$ and T. porosum TPST6 were able to produce high amounts of lipid between $24.5 \%$ and $34.1 \%$ lipid per dry biomass in both cultivation methods. In contrast, $P$. segobiensis SSOH12 with just $11.2 \%$ lipid per dry biomass could not be classified as oleaginous yeast when cultured in shake flasks with the chosen medium. However, when cultured in a $2.5 \mathrm{~L}$-bioreactor $24.6 \%$ lipid per dry biomass was realized with $P$. segobiensis SSOH12, which corresponds to twice the amount obtained with shake flask cultivation. Low aeration rates in shake flasks may be the reason for low lipid level since increasing cell densities lead to less available oxygen. Moreover, a nitrogen limiting mineral salt medium with adequate buffering capacities would be more appropriate for shake flask cultures. Under low aeration rates facultative anaerobic strains start to produce ethanol. It is thought that the oxygen limitation during the maximal growth phase in the cultivations with bioreactors occurred as the agitation speed was too low for the present cell density. In the present study

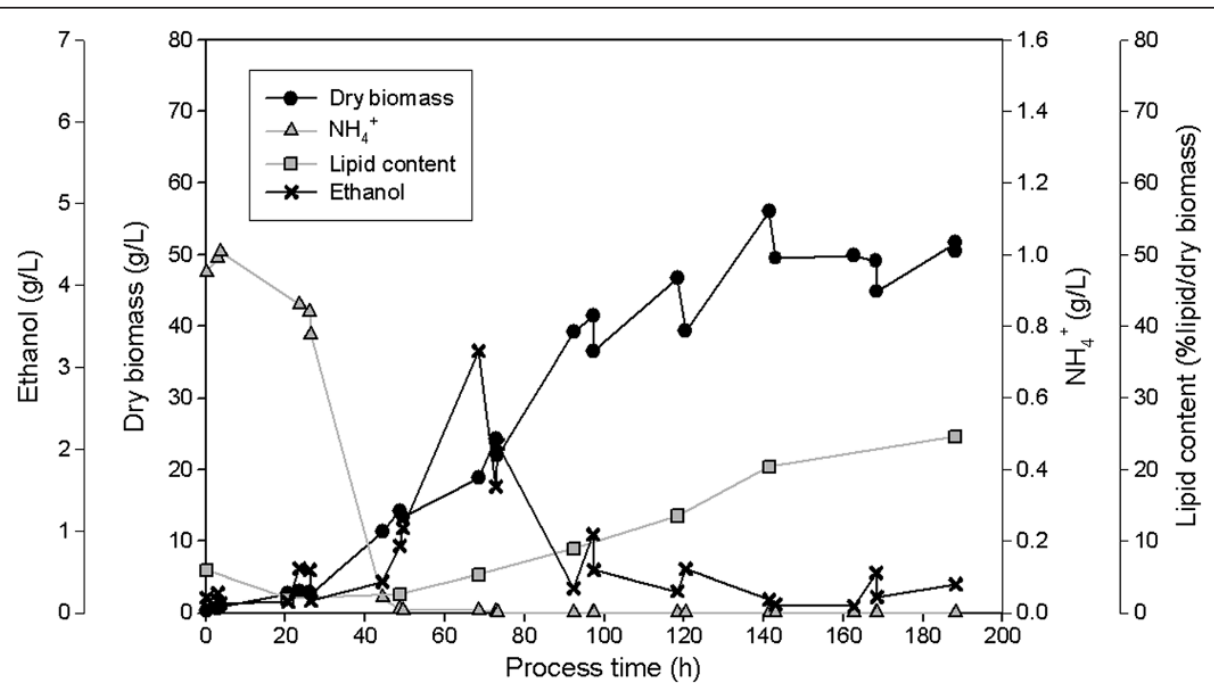

Figure $6 \mathrm{SSOH} 12$ cultivated on glucose in $2.5 \mathrm{~L}$-bioreactor in mineral salt medium at $\mathrm{pH} 5$ and $25^{\circ} \mathrm{C}$; glucose was fed daily. $\mathrm{NH}_{4}^{+}$consumption, production of biomass, lipid and ethanol are presented. 
Table 3 Fatty acid profile of the three isolates SSOH12, CPOH4 and TPST6 with respect to chosen carbon source employed in growth medium; amounts of fatty acids are given in\% on total fatty acids; fatty acids detected with amounts less than $1 \%$ are combined to "Trace fatty acids"

\begin{tabular}{|c|c|c|c|c|c|c|c|c|}
\hline \multirow[t]{2}{*}{ Isolate } & \multirow[t]{2}{*}{ Carbon source } & \multicolumn{7}{|c|}{ Type of fatty acid (\% of total fatty acids) } \\
\hline & & C16:0 & C16:1 & C18:0 & C18:1 & C18:2 & C18:3 & Trace fatty acids \\
\hline $\mathrm{SSOH} 12$ & Glucose & 19.1 & 16.0 & 2.0 & 51.8 & 7.5 & 0.5 & 3.1 \\
\hline \multirow[t]{2}{*}{$\mathrm{CPOH} 4$} & Glucose & 18.4 & 0.3 & 5.3 & 59.4 & 8.7 & 0.9 & 7.0 \\
\hline & Xylose & 20.1 & 0.4 & 4.7 & 55.1 & 11.1 & 1.1 & 7.5 \\
\hline \multirow[t]{2}{*}{ TPST6 } & Glucose & 19.5 & 0.3 & 17.0 & 40.4 & 17.8 & 1.3 & 3.7 \\
\hline & Xylose & 21.1 & 0.3 & 15.5 & 39.6 & 18.7 & 1.0 & 3.8 \\
\hline
\end{tabular}

P. segobiensis SSOH12 produced ethanol as by-product as soon as the dissolved oxygen level reached $0 \%$ (data not shown). Hence, this strain may be classified as a facultative anaerobic yeast. This shows the importance of adequate aeration even for screening experiments. To prevent the production of ethanol and to increase the production of lipid by higher dissolved oxygen the agitation speed has to be increased. Further experiments should be done with controlled dissolved oxygen. Strains that gave a positive test result with Sudan Black B staining on agar plates, but a negative result in shake flasks with YM medium supplemented with glucose, are worthwhile to examine in further detail in a well aerated bioreactor as not only the nitrogen limitation but also the sufficient oxygen supply may be a prerequisite for lipid production.

In addition to the oxygenation rate, the $\mathrm{pH}$ of the medium is also an important parameter to consider during the screening of lipid producing strains as the $\mathrm{pH}$ value cannot be controlled in shake flasks. Most strains are acid labile and would not grow well in acidic environment like Candida shehatae (Kastner et al. 1996), and therefore would even not produce any favored lipid. The shake flask cultivation of C. curvatus and C. podzolicus $\mathrm{CPOH} 4$ in mineral salt medium (data not shown) resulted in a decrease of the $\mathrm{pH}$ value from 5.0 to 2.0 within 60 hours. The well known oleaginous yeast $C$. curvatus produced just a maximum of $15 \%$ lipid per dry biomass under these conditions whereas C. podzolicus $\mathrm{CPOH} 4$ ended up with 23\% lipid within $100 \mathrm{~h}$ cultivation time (data not shown). The advantage of $C$. podzolicus $\mathrm{CPOH} 4$ is its acid resistance, which is beneficial in podsol soil, an acidic environment, from which $C$. podzoilicus $\mathrm{CPOH} 4$ could be isolated and from which its name arose (Botes et al. 2005). Cultivating $C$. curvatus in $\mathrm{YM}$ medium the $\mathrm{pH}$ decreased only from 5 to 4 and yielded up to more than $40 \%$ lipid per dry biomass. This shows that the YM medium supplemented with glucose has good buffering conditions and is a useful medium for a first examination of yeast strains.

\section{Characterization of newly isolated strains}

SSOH12 was identified as Pichia segobiensis. This strain belongs to the ascomycetes. The second highest agreement of SSOH12 was with Scheffersomyces stipitis, also known as Pichia stipitis. Pichia stipitis (Nigam 2001) and Scheffersomyces stipitis (Liu et al. 2010) are well described within the context of microbial ethanol production, but have not been mentioned previously with regards to lipid production. Furthermore they are able to assimilate hemicellulosic compounds (Ferreira et al. 2011; Nigam 2001).

Aside from the focus on the production of ethanol under anaerobic conditions, this study has shown that $P$. segobiensis SSOH12 is able to produce more than $24 \%$ lipid content under aerobic conditions and sufficient oxygenation. As the ethanol production of P. segobiensis SSOH12 commences only after oxygen limitation occurs, the crabtree effect like in Saccharomyces cerevisiae (Al-mhanna 2010) is excluded. This could be confirmed for the closely related yeast, Scheffersomyces stipitis (Papini et al. 2012). Under aerobic conditions this yeast can be classified as oleaginous yeast and process optimization with higher dissolved oxygen level might increase the lipid yield.

The special feature of $P$. segobiensis SSOH12 concerning the fatty acid profile is the different composition to most other oleaginous yeast containing a fatty acid similar to cacao-butter with the main components of C16:0, C18:0, C18:1 and C18:2. In addition, P. segobiensis SSOH12 comprises 16\% palmitoleic acid (C16:1), an omega-7 mono-unsaturated fatty acid which has been shown to have positive effects against obesity (Yang et al. 2011) and potential for the prevention of brain and cardiovascular diseases (Matsunaga et al. 1995). It is a component of some oil seeds, especially sea-buckthorn (Fatima et al. 2012) or macadamia (Nestel et al. 1994). An alternative source via microbial production is the opportunity to produce palmitoleic acid in sufficient quantities for possible future applications in medicine. P. segobiensis SSOH12 has been described as one of the best xylose-converting strains (Liu et al. 2010; Toivola et al. 1984). Hence, the investigation of lipid production with xylose as the carbon source may be a worthwhile exercise.

The two other isolated strains were identified as Cryptococcus podzolicus (CPOH4) and Trichosporon porosum 
(TPST6). Both strains belong to the yeast-like anamorphic basidiomycetes and are found in soil (Botes et al. 2005; Colombo et al. 2011). They are also known to assimilate hemicelluloses (Middelhoven et al. 2001; Shubakov 2000).

Trichosporon sp. and Cryptococcus sp. in general are known to belong to the oleaginous strains (Gujjari et al. 2011; Zhu et al. 2008; Hu et al. 2011), whereas T. porosum and C. podzolicus have not been mentioned before in relation to microbial oil production. The results of this study reveal that both strains are able to produce at least 30\% lipid per dry biomass when grown on glucose or xylose as carbon source. Therefore, C. podzolicus CPOH4 and T. porosum TPST6 can be characterized for the first time as oleaginous yeasts. $T$. porosum TPST6 produced in this study almost $20 \%$ linolic acid which makes it unique among other Trichosporon species which generally yield less than $10 \%$, e.g. Trichosporon fermentans less than 8\% (Huang et al. 2012) and Trichosporon cutaneum less than $3.4 \%$ (Hu et al. 2011).

The first assumption to explain the observed acid production in the culture broth of $\mathrm{CPOH} 4$ and TPST6 was that excessive citric acid may be secreted into the medium which serves as a precursor for Acetyl-CoA and further for the production of triacylglycerides in oleaginous strains (Ratledge 2002). However, no citric acid could be determined in this study. Instead, gluconic acid was measured as additional by-product with high concentrations up to $30 \mathrm{~g} / \mathrm{L}$ for $C$. podzolicus $\mathrm{CPOH} 4$ and $12 \mathrm{~g} / \mathrm{L}$ for $T$. porosum TPST6. Both strains are simultaneous producers of lipid and gluconic acid.

Gluconic acid and its derivates find wide application in the food and pharmaceutical industries. Therefore it could be worthwhile to improve gluconic acid production with the newly isolated yeasts $C$. podzolicus $\mathrm{CPOH} 4$ or T. porosum TPST6. The ascomycete Aspergillus niger (Ramachandran et al. 2006) and yeast like Aureobasidium pullulans (Anastassiadis and Rehm 2006) are other examples of gluconic acid producers with high production rates of $120-140 \mathrm{~g} / \mathrm{L}$ and up to $370 \mathrm{~g} / \mathrm{L}$, respectively.

C. podzolicus $\mathrm{CPOH} 4$ and $T$. porosum TPST6 give the opportunity to favor either the production of intracellular oil or the production of gluconic acid or rather the simultaneous production. An advantage of the simultaneous production of lipid and gluconic acid would be the easy separation of both products as the oil is produced intracellular, whereas the gluconic acid is secreted into the culture broth. Higher aeration rates to prevent oxygen limitation are required in any case to increase the product levels, although ethanol production is negligible. Negligible ethanol production which started just at the stage of oxygen limitation verifies that both strains are crabtree negative. To avoid the production of gluconic acid as by-product in a lipid production process, xylose may be the carbon source of choice. However, another interesting approach could be the combined feeding of glucose and xylose as both carbon sources are components of hydrolyzed wood and straw waste. If glucose and xylose were consumed simultaneously hydrolyzed straw and wood wastes could be used as low-cost carbon source. Trichosporon cutaneum (Hu et al. 2011) and Cryptococcus curvata (Heredia and Ratledge 1988) could be described as such strains. The use of other low-cost carbon sources and further process optimization to increase the lipid yield are further possible approaches.

Both isolates $C$. podzolicus $\mathrm{CPOH} 4$ and $T$. porosum TPST6 are suitable for a lipid production bioprocess, but $T$. porosum TPST6 shows a more interesting fatty acid profile with $18.7 \%$ linoleic acid (C18:2). Moreover, xylose as carbon source favours the lipid production whereas glucose as carbon source leads to a simultaneous production of gluconic acid and intracellular lipid.

P. segobiensis is worthwhile to be further investigated because of its considerable amount (16\%) of plamitoleic acid (C16:1) which may be suitable for medical applications.

\section{Competing interests}

The authors declare that they have no competing interests.

\section{Acknowledgements}

This work was funded by the "Bundesministerium für Wirtschaft und Technologie" within the ERA SME project BiCycle Integrated new concept(s) for the production of Single Cell Oils (SCO's) on an economic scale in cooperation with the companies EnBW AG, EVONIK Industries AG, B.R.A.I.N AG and Phytowelt Green Technologies $\mathrm{GmbH}$.

Received: 30 December 2013 Accepted: 3 January 2014 Published online: 18 March 2014

\section{References}

Al-mhanna MNM (2010) Observation of Crabtree effect and diauxic behaviour of yeast by using absorption. Chem Eng Trans 21:1465-1470, doi:10.3303/ CET1021245

Anastassiadis S, Rehm H-J (2006) Continuous gluconic acid production by the yeast-like Aureobasidium pullulans in a cascading operation of two bioreactors. Appl Microbiol Biotechnol 73(3):541-548, doi:10.1007/s00253-006-0499-y Botes AL, Lotter J, Rhode OHJ, Botha A (2005) Interspecies differences in the enantioselectivity of epoxide hydrolases in Cryptococcus laurentii (Kufferath) C.E. skinner and Cryptococcus podzolicus (Bab'jeva \& Reshetova) Golubev. Syst Appl Microbiol 28(1):27-33, doi:10.1016/j.syapm.2004.10.003

Colombo AL, Padovan ACB, Chaves GM (2011) Current Knowledge of Trichosporon spp. and Trichosporonosis. Clin Microbiol Rev 24(4):682-700

Evans CT, Ratledge C, Gilbert SC (1985) A rapid screening method for lipid accumulating yeast using a replica printing technique. J Microbiol Methods 4(3-4):203-210

Fatima T, Snyder CL, Schroeder WR, Cram D, Datla R, Wishart D, Weselake RJ, Krishna P (2012) Fatty acid composition of developing sea buckthorn (Hippophae rhamnoides L.) berry and the transcriptome of the mature seed. PLoS ONE 7(4):e34099, doi:10.1371/journal.pone.0034099

Ferreira AD, Mussatto SI, Cadete RM, Rosa CA, Silva SS (2011) Ethanol production by a new pentose-fermenting yeast strain, Scheffersomyces stipitis UFMG-IMH 43.2, isolated from the Brazilian forest. Yeast 28(7):547-554, doi:10.1002/ yea.1858

Fujita SI, Senda Y, Nakaguchi S, Hashimoto T (2001) Multiplex PCR using internal transcribed spacer 1 and 2 regions for rapid detection and identification of yeast strains. J Clin Microbiol 39(10):3617-3622, doi:10.1128/ jem.39.10.3617-3622.2001

Griffiths MJ, Harrison STL (2009) Lipid productivity as a key characteristic for choosing algal species for biodiesel production. J Appl Phycol 21(5):493-507, doi:10.1007/s10811-008-9392-7 
Gujjari P, Suh S-O, Coumes K, Zhou JJ (2011) Characterization of oleaginous yeasts revealed two novel species: Trichosporon cacaoliposimilis sp nov and Trichosporon oleaginosus sp nov. Mycologia 103(5):1110-1118, doi:10.3852/10-403

Heredia L, Ratledge C (1988) Simultaneous utilization of glucose and xylose by Candida curvata D in continuous culture. Biotechnol Lett 10(1):25-30

Hu C, Wu S, Wang Q, Jin G, Shen H, Zhao ZK (2011) Simultaneous utilization of glucose and xylose for lipid production by Trichosporon cutaneum. Biotechnol Biofuels 4(25):25, doi:10.1186/1754-6834-4-25

Huang C, Wu H, Liu ZJ, Cai J, Lou WL, Zong MH (2012) Effect of organic acids on the growth and lipid accumulation of oleaginous yeast Trichosporon fermentans. Biotechnol Biofuels 5(4):4, doi:10.1186/1754-6834-5-4

Kastner JR, Roberts RS, Jones WJ (1996) Effect of pH on cell viability and product yields in D-xylose fermentations by Candida shehatae. Appl Microbiol Biotechnol 45(1-2):224-228

Lee H, Biely P, Latta RK, Barbosa MFS, Schneider H (1986) Utilization of xylan by yeasts and its conversion to ethanol by Pichia stipitis strains. Appl Environ Microbiol 52(2):320-324

Li Q, Du W, Liu DH (2008) Perspectives of microbial oils for biodiesel production Appl Microbiol Biotechnol 80(5):749-756, doi:10.1007/s00253-008-1625-9

Liu T, Zou W, Liu L, Chen J (2010) A constraint-based model of Scheffersomyces stipitis for improved ethanol production. Biotechnol Biofuels 5(72):72, doi:10.1186/1754-6834-5-72

Matsunaga T, Takeyama H, Miura Y, Yamazaki T, Furuya H, Sode K (1995) Screening of marine cyanobacteria for high palmitoleic acid production. FEMS Microbiol Lett 133(1-2):137-141, doi:10.1016/0378-1097(95)00350-e

Meesters PEAP, Huijberts GNM, Eggink G (1996) High cell density cultivation of the lipid accumulating yeast Cryptococcus curvatus using glycerol as a carbon source. Appl Microbiol Biotechnol 45(5):575-579

Middelhoven WJ, Scorzetti G, Fell JW (2001) Trichosporon porosum comb. nov. an anamorphic basidiomycetous yeast inhabiting soil, related to the loubieri/ laibachii group of species that assimilate hemicelluloses and phenolic compounds. FEMS Yeast Res 1(1):15-22, doi:10.1111/j.1567-1364.2001. tb00009.x

Nestel P, Clifton P, Noakes M (1994) Effects of increasing dietary palmitoleic acid compared with palmitic and oleic acids on plasma lipids of hypercholesterolemic men. J Lipid Res 35(4):656-662

Nigam JN (2001) Development of xylose-fermenting yeast Pichia stipitis for ethanol production through adaptation on hardwood hemicellulose acid prehydrolysate. J Appl Microbiol 90(2):208-215, doi:10.1046/j.1365-2672.2001.01234.x

Pan LX, Yang DF, Li S, Wei L, Chen GG, Liang ZQ (2009) Isolation of the oleaginous yeasts from the soil and studies of their lipid producing capacities. Food Technol Biotechnol 47(2):215-220

Papanikolaou S, Aggelis G (2011) Lipids of oleaginous yeasts. Part I: biochemistry of single cell oil production. Eur J Lipid Sci Technol 113(8):1031-1051, doi:10.1002/ejlt.201100014

Papini M, Nookaew I, Uhlen M, Nielsen J (2012) Scheffersomyces stipitis: a comparative systems biology study with the Crabtree positive yeast Saccharomyces cerevisiae. Microb Cell Fact 11:136, doi:10.1186/1475-2859-11-136

Ramachandran S, Fontanille P, Pandey A, Larroche C (2006) Gluconic acid: properties, applications and microbial production. Food Technol Biotechnol 44(2):185-195

Ratledge C (1991) Microorganisms for lipids. Acta Biotechnol 11(5):429-438

Ratledge C (1993) Single cell oils - have they a biotechnological future? Trends Biotechnol 11(7):278-284

Ratledge C (2002) Regulation of lipid accumulation in oleaginous microorganisms. Biochem Soc Trans 3(6):1047-1050

Shubakov AA (2000) The beta-xylosidase production by yeast Cryptococcus podzolicus. Bioorg Khim 26(8):613-616

Thiru M, Sankh S, Rangaswamy V (2011) Process for biodiesel production from Cryptococcus curvatus. Bioresour Technol 102(22):10436-10440, doi:10.1016/j. biortech.2011.08.102

Toivola A, Yarrow D, Vandenbosch E, Vandijken JP, Scheffers WA (1984) Alcoholic fermentation of deuterium xylose by yeasts. Appl Environ Microbiol 47(6):1221-1223

Vorapreeda T, Thammarongtham C, Cheevadhanarak S, Laoteng K (2012) Alternative routes of acetyl-CoA synthesis identified by comparative genomic analysis: involvement in the lipid production of oleaginous yeast and fungi. Microbiology 158:217-228, doi:10.1099/mic.0.051946-0

Ward OP, Singh A (2005) Omega-3/6 fatty acids: alternative sources of production. Process Biochem 40(12):3627-3652, doi:10.1016/j.procbio.2005.02.020
Yang ZH, Miyahara H, Hatanaka A (2011) Chronic administration of palmitoleic acid reduces insulin resistance and hepatic lipid accumulation in KK-A(y) mice with genetic type 2 diabetes. Lipids Health Dis 10:8, doi:12010.1186/ $1476-511 x-10-120$

Zhang J, Fang X, Zhu XL, Li Y, Xu HP, Zhao BF, Chen L, Zhang XD (2011) Microbial lipid production by the oleaginous yeast Cryptococcus curvatus $\mathrm{O} 3$ grown in fed-batch culture. Biomass Bioenergy 35(5):1906-1911, doi:10.1016/ j.biombioe.2011.01.024

Zhu LY, Zong MH, Wu H (2008) Efficient lipid production with Trichosporon fermentans and its use for biodiesel preparation. Bioresour Technol 99(16):7881-7885, doi:10.1016/j.biortech.2008.02.033

doi:10.1186/s13568-014-0024-0

Cite this article as: Schulze et al:: Characterization of newly isolated oleaginous yeasts - Cryptococcus podzolicus, Trichosporon porosum and Pichia segobiensis. AMB Express 2014 4:24.

\section{Submit your manuscript to a SpringerOpen ${ }^{\odot}$ journal and benefit from:}

- Convenient online submission

- Rigorous peer review

- Immediate publication on acceptance

- Open access: articles freely available online

- High visibility within the field

- Retaining the copyright to your article

Submit your next manuscript at $>$ springeropen.com 